\title{
Improving survival in ACLF: growing evidence for use of G-CSF
}

\author{
D. A. Simonetto ${ }^{1}$ V. H. Shah ${ }^{1}$ P. S. Kamath ${ }^{1}$
}

Received: 5 October 2017/ Accepted: 2 November 2017/Published online: 9 November 2017

(C) Asian Pacific Association for the Study of the Liver 2017

Acute-on-chronic liver failure (ACLF) has recently been proposed as an alternate route in the natural history of patients with chronic liver disease, and is associated with extremely high mortality. ACLF is characterized by acute hepatic decompensation in a patient with previously diagnosed or undiagnosed chronic liver disease [1] and failure of one or more extrahepatic organ [2]. Currently, there are no specific therapies for ACLF; management focuses on addressing the precipitant factor and supportive care. Liver transplantation is the ultimate treatment for those who are acceptable candidates, but is limited by organ shortage and high frequency of contraindications in this group of patients. Granulocyte-colony stimulating factor (G-CSF) has recently been proposed as a candidate ACLF treatment. G-CSF promotes mobilization of hematopoietic stem cells and proliferation of hepatic progenitor cells in animal models of liver failure [3] as well as in patients with alcoholic hepatitis, a common form of ACLF in the Western world [4]. A few small randomized clinical trials have demonstrated not only improvement in liver function with G-CSF but also significant survival benefit compared with standard medical therapy for ACLF [5-8]. However, additional randomized trials in different populations are needed to corroborate these findings before widespread use of G-CSF can be advocated worldwide.

In this issue of Hepatology International, Sarin and colleagues report the effects of G-CSF treatment in addition to standard medical therapy in patients with ACLF in a tertiary hospital in Bangladesh. The authors randomized 32

P. S. Kamath

Kamath.patrick@mayo.edu

1 Mayo Clinic Gastroenterology and Hepatology, 200 First St. S.W., Rochester, MN 55905, USA patients with diagnosis of ACLF according to the criteria proposed by the Asian Pacific Association for the Study of the Liver (APASL) [1]. The most common underlying liver disease was chronic viral hepatitis B (HBV) infection, and HBV reactivation was the most common precipitant of ACLF (in 19 of 32 patients). Other causes included acute hepatitis $\mathrm{E}$ infection and drug-induced liver injury (herbals and antitubercular drug). No cases of alcoholic hepatitis were included. The patients were randomized to receive G-CSF at $5 \mathrm{mcg} / \mathrm{kg} / \mathrm{day}$ subcutaneously for 6 days versus standard medical therapy, and were followed for 90 days. G-CSF led to significant reduction of Child-Turcotte-Pugh (CTP) score at 90 days compared with conventional therapy. Model for End-Stage Liver Disease (MELD) score was also reduced, but did not reach statistical significance. More importantly, G-CSF significantly improved survival at 90 days (87.5 versus $50 \%$ in control group). No mortality difference was found at 30 days.

The findings of Sarin and colleagues are consistent with, albeit not as robust as, those of prior studies and support a role for G-CSF in management of ACLF. A potential explanation for these differences may lie in the dual mechanistic benefits of G-CSF and the populations studied. In addition to promoting hepatic regeneration through stem cell mobilization, G-CSF may also reduce risk of infection. A previous study showed significant reduction in risk of septic shock in decompensated cirrhotic patients treated with G-CSF in combination with erythropoietin (38.5 versus $6.9 \%$ ) [9]. The same group had previously demonstrated that G-CSF may restore the immune dysfunction observed in ACLF, through an increase in circulating and intrahepatic myeloid and plasmacytoid dendritic cells [10]. This additional benefit of G-CSF may be particularly important in patients with alcoholic hepatitis, given their increased risk of bacterial infection [11]. 
Etiologies of ACLF differ by region, with reactivation of hepatitis B and superimposed acute hepatitis E more common in Asia, whereas alcoholic hepatitis and sepsis are reportedly more common in Western hospitals. Previous trials had mainly included patients with ACLF caused by alcoholic hepatitis, in whom G-CSF demonstrated a significant reduction in CTP and MELD scores and markedly improved 30-, 60-, and 90-day survival compared with standard medical care $[6,7]$. A short report also recently showed a potential role of plasmapheresis with plasma exchange combined with G-CSF for alcoholic hepatitisrelated ACLF. The authors suggested that plasma exchange may promote a more favorable hepatic microenvironment for the effect of G-CSF, although this remains speculative [12]. To date, no studies have been carried out in patients with ACLF and sepsis, but it is possible that these patients might have the greatest benefit with G-CSF therapy.

A few limitations of the present study should be mentioned. The beneficial effects of G-CSF have previously been shown to be dose dependent, and prior studies have used dosages as high as $15 \mathrm{mcg} / \mathrm{kg} /$ day for as long as 12 days, with a low rate of adverse events reported. Therefore, the optimal dose and duration of G-CSF treatment still need to be determined, and may depend on the etiology of ACLF. The authors did not report the rate of bacterial infection, and the reported causes of death are not well defined. The main drivers of mortality in ACLF are septic shock and multiorgan or hepatic failure, both of which can potentially be prevented or reversed with G-CSF. In this study, however, the reported causes of death include hepatorenal syndrome and electrolyte disturbances, and it is unclear how this complication was treated. Also, the rates of respiratory or circulatory failure, which are not part of the MELD or CTP score, were not reported. Inclusion of sequential organ failure assessment (SOFA) or chronic liver failure (CLIF)-SOFA scores would have been helpful to assess the severity of multiorgan failure in this cohort of patients. The authors also missed an opportunity to study the effect of G-CSF in a homogeneous population of patients with ACLF secondary to reactivation of HBV infection. The three patients without HBV infection could have been excluded; arguably, even the patients with underlying HBV infection with precipitating factors related to herbal infection, antitubercular drugs, and hepatitis E virus (HEV) infection should also have been excluded. Given the heterogeneous group, the study is too small to conclude that G-CSF may be used in ACLF due only to HBV reactivation.

In summary, the work of Sarin and colleagues strengthens the evidence supporting use of G-CSF for treatment of ACLF. While the magnitude of the benefit of G-CSF may vary according to the cause of ACLF, improvement in survival has been consistently

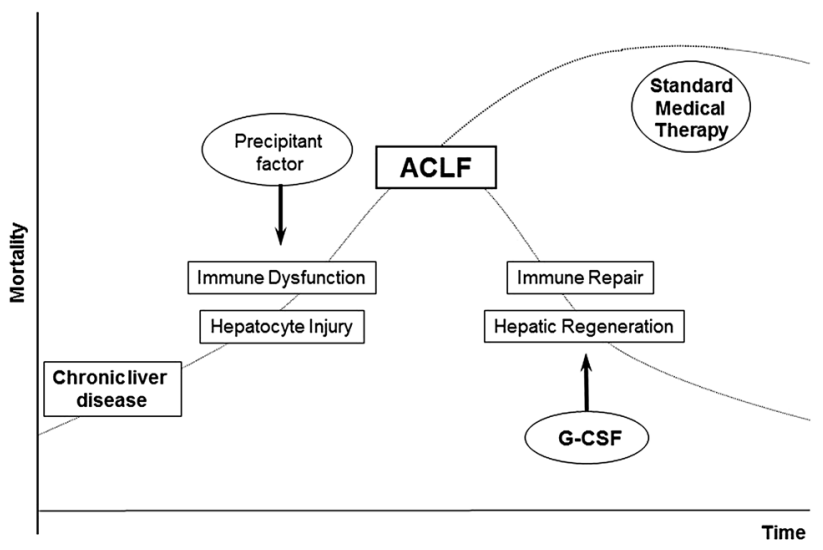

Fig. 1 Role of G-CSF in management of ACLF and its possible effects on the immune system and hepatic regeneration

demonstrated. This effect is likely secondary to restoration of the impaired immune system in ACLF, thereby reducing risk of infection, and mobilization of stem cells promoting hepatic regeneration. At present, more studies are needed to determine which groups of ACLF patients, based on severity and etiology, will benefit from G-CSF therapy and to what degree. Larger multicenter double-blind randomized trials in homogeneous patient groups are the next step in validating the role and potential mechanisms of action of G-CSF in ACLF (Fig. 1).

\section{Compliance with ethical standards}

Conflict of interest D. A. Simonetto, V. H. Shah, and P. S. Kamath declare that they have no conflicts of interest.

Funding This work was supported by NIH Grant no. AA 21788 from the National Institute on Alcohol Abuse and Alcoholism (NIAAA).

\section{References}

1. Sarin SK, Kedarisetty CK, Abbas Z, Amarapurkar D, Bihari C, Chan $\mathrm{AC}$, et al. Acute-on-chronic liver failure: consensus recommendations of the Asian Pacific Association for the Study of the Liver (APASL) 2014. Hepatol Int 2014;8:453-471

2. Jalan R, Yurdaydin C, Bajaj JS, Acharya SK, Arroyo V, Lin HC, et al. Toward an improved definition of acute-on-chronic liver failure. Gastroenterology 2014;147:4-10

3. Theocharis SE, Papadimitriou LJ, Retsou ZP, Margeli AP, Ninos SS, Papadimitriou JD. Granulocyte-colony stimulating factor administration ameliorates liver regeneration in animal model of fulminant hepatic failure and encephalopathy. Dig Dis Sci 2003;48:1797-1803

4. Spahr L, Lambert JF, Rubbia-Brandt L, Chalandon Y, Frossard JL, Giostra E, et al. Granulocyte-colony stimulating factor induces proliferation of hepatic progenitors in alcoholic steatohepatitis: a randomized trial. Hepatology 2008;48:221-229

5. Duan XZ, Liu FF, Tong JJ, Yang HZ, Chen J, Liu XY, et al. Granulocyte-colony stimulating factor therapy improves survival in patients with hepatitis B virus-associated acute-on-chronic liver failure. World J Gastroenterol 2013;19:1104-1110 
6. Garg V, Garg H, Khan A, Trehanpati N, Kumar A, Sharma BC, et al. Granulocyte colony-stimulating factor mobilizes CD34(+) cells and improves survival of patients with acute-on-chronic liver failure. Gastroenterology 2012;142(505-512):e501

7. Singh V, Sharma AK, Narasimhan RL, Bhalla A, Sharma N, Sharma R. Granulocyte colony-stimulating factor in severe alcoholic hepatitis: a randomized pilot study. Am J Gastroenterol 2014;109:1417-1423

8. Di Campli C, Zocco MA, Saulnier N, Grieco A, Rapaccini G, Addolorato G, et al. Safety and efficacy profile of G-CSF therapy in patients with acute on chronic liver failure. Dig Liver Dis 2007;39:1071-1076

9. Kedarisetty CK, Anand L, Bhardwaj A, Bhadoria AS, Kumar G, Vyas AK, et al. Combination of granulocyte colony-stimulating factor and erythropoietin improves outcomes of patients with decompensated cirrhosis.

Gastroenterology 2015;148(1362-1370):e1367

10. Khanam A, Trehanpati N, Garg V, Kumar C, Garg H, Sharma $\mathrm{BC}$, Sarin SK. Altered frequencies of dendritic cells and IFNgamma-secreting $\mathrm{T}$ cells with granulocyte colony-stimulating factor (G-CSF) therapy in acute-on-chronic liver failure. Liver Int 2014;34:505-513

11. Gustot T, Fernandez J, Szabo G, Albillos A, Louvet A, Jalan R, et al. Sepsis in alcohol-related liver disease. J Hepatol 2017;67(5):1031-1050

12. Piscaglia AC, Arena V, Passalacqua S, Gasbarrini A. A case of granulocyte-colony stimulating factor/plasmapheresis-induced activation of granulocyte-colony stimulating factor-positive hepatic progenitors in acute-on-chronic liver failure. Hepatology 2015;62:649-652 